\title{
Pneumococcal arthritis mimicking juvenile chronic arthritis
}

\author{
A EARLEY,${ }^{*}$ S RICHMAN, $\dagger$ AND B M ANSELL $\ddagger$ \\ Department of Paediatrics, *Northwick Park Hospital, Harrow and †Central Middlesex Hospital, London, \\ and $\ddagger$ Division of Rheumatology, Clinical Research Centre, Harrow, Middlesex
}

SUMMARY Two young children referred as pauciarticular juvenile chronic arthritis were subsequently found to have pneumococcal septic arthritis.

Failure to diagnose septic arthritis can lead to serious joint damage, but it may not be easy to exclude. Morrisey and Shore, recorded 10 out of 32 cases of septic arthritis wrongly diagnosed in the first instance. ${ }^{1}$ We report two children whose initial investigations for septic arthritis were negative, but who had persistent symptoms and had been diagnosed as juvenile chronic arthritis.

\section{Case reports}

The children, both boys, aged 20 months and 3 years, were referred for management of pauciarticular juvenile chronic arthritis. Both gave a three to four week history of swelling of one knee with refusal to bear weight; one child also had transient swelling of an ankle. A five day course of oral antibiotics for a sore throat had been given to the older child before the onset of the knee problem. The younger child had had pneumococcal pneumonia six months earlier. Both children had had sevieral sets of negative blood cultures at the referring hospitals and were being managed with non-steroidal anti-inflammatory drugs.

On presentation both children had an acutely tender hot swollen knee joint that was held firmly in flexion. Although refusing to move the joint neither child was acutely toxic or ill. One developed a spike of fever and more pain on stopping the non-steroidal drug. Both children were anaemic, with haemoglobin concentrations of 75 and $96 \mathrm{~g} / \mathrm{l}$, white cell counts were normal, but the erythrocyte sedimentation rates in the first hour were 100 and $95 \mathrm{~mm}$. Aspiration of the joints showed turbid fluid with a high polymorph count. This fluid grew Streptococcus pneumoniae (sensitive to benzylpenicillin) in one child. The other boy, who had received antibiotics before admission, had sterile joint fluid, but a blood culture taken at the same time as joint aspiration yielded the organism. Both children were managed with six weeks of benzylpenicillin and appropriate physiotherapy and splinting and made a complete recovery. Further immunological investigation of the child with previous pneumococcal infection gave normal results.

\section{Discussion}

Both these children had had a swollen joint for four weeks. The striking clinical feature was the exquisitely tender joint and its immobility. Basic investigations showing a very high erythrocyte sedimentation rate and a low haemoglobin concentration are unlikely in pauciarticular juvenile chronic arthritis. The state of the joint and these laboratory features were indications for aspiration of the joint.

Antibiotics, however briefly administered, affect the growth of organisms from blood and joint fluid, and repeated cultures should be taken if a joint remains hot and tender. Despite repeated attempts, up to $40 \%$ of synovial fluid cultures are uninformative $^{2}$ and here culture of blood or other site of local infection may help.

Failure to grow the organism from the joint in one child raises the question of whether the arthritis was 'reactive' in this patient. Reactive arthritis has been reported after pneumococcal meningitis, ${ }^{3}$ although is much more commonly described after haemophilus and meningococcal infections. The mechanism is presumed to be an immunological one with an inflammatory response to bacterial cell wall products rather than live organisms in the joint. ${ }^{2}$ Cases of reactive arthritis after bacterial sepsis that were reported by Rush et al did not respond to antibiotics, ${ }^{4}$ whereas our patient responded dramatically to benzylpenicillin. Taking this clinical response with the positive blood culture at the time of joint aspiration, we feel justified in also calling this case septic arthritis.

In one child the presence of another swollen joint was misleading, but transient synovitis occurs in more than one joint in up to $10 \%$ of cases of septic arthritis or osteomyelitis. Non-steroidal drugs were effective in suppressing constitutional signs of sepsis in one child and thus tended to mask the clinical picture of septic arthritis.

We thank Drs AM Mobarak and B Taylor for permission to report their patients, and $\mathrm{Mr}$ CM McCullough who performed the joint aspirations. 
References
1 Morrisey RT, Shore SL. Bone and joint sepsis. Pediatr Clin
North Am 1986;33:1551-64.
2 Anonymous. Bacterial arthritis. Lancet 1986;ii:721-2.
${ }^{3}$ Golden SE, Kelly JC. Pneumococcal meningitis complicated by
immune complex-mediated arthritis. Am J Dis Child 1987;141:
603-4.
${ }^{4}$ Rush PJ, Shore A, Inman R, et al. Arthritis associated with
Haemophilus influenzae meningitis: septic or reactive? J Pediat 1986;109:412-5.

Correspondence to Dr A Earley, Department of Paediatrics, Northwick Park Hospital, Watford Road, Harrow, Middlesex HA1 $3 \mathrm{UJ}$.

Accepted 18 April 1988

\title{
Growth hormone, suspected gonadotrophin deficiency, and ring 18 chromosome
}

\author{
S S ABUSREWIL, ${ }^{*}$ A McDERMOTT, $\dagger$ AND D C L SAVAGE* \\ ${ }^{*}$ Bristol Children's Hospital and †South Western Regional Cytogenetics Service, Southmead Hospital, Bristol
}

SUMMARY A boy with a ring 18 chromosome karyotype was referred because of short stature; he had growth hormone deficiency and possible hypogonadotrophic hypogonadism. Many children with major chromosomal abnormalities are short, but this case emphasises the need to consider growth hormone deficiency in addition.

About 60 patients with a ring 18 chromosome karyotype have been reported. ${ }^{1}$ Phenotypically they are short and obese with mild dysmorphic features including hypertelorism, epicanthic folds, slight micrognathia, and small hands with short, tapering fingers. Boys may have a small penis and cryptorchism. All have pronounced retardation of growth and there have been two reports of additional growth hormone deficiency. ${ }^{23}$ We report a child with growth hormone deficiency and possible gonadotrophin deficiency.

\section{Case report}

The boy was born to healthy non-consanguinous parents at 40 weeks' gestation weighing $4900 \mathrm{~g}$. $\mathrm{He}$ had a low Apgar score and resuscitation included a brief period of intubation. His development was delayed and at 3.5 years he was noted to be short (height $-3 \mathrm{SD}$ ) and comparatively obese (weight 10 th centile), with an unusual facies. Examination of the peripheral lymphocytes showed an abnormal male karyotype of 46 chromosomes, including one ring 18 chromosome. The precise breakpoints in the formation of the ring chromosome could not be determined, but the morphology and stability of the ring implied that the deleted distal segments were comparatively small. The parents' chromosomes were normal.
At the age of 12 he was referred to the growth clinic because of pronounced retardation of growth and obesity. His height was $112 \mathrm{~cm}(-4.8 \mathrm{SD})$, and he weighed $37 \mathrm{~kg}$ (50th centile). He had several of the dysmorphic features that are described in children with ring 18 karyotype including a small penis $(<2 \mathrm{~cm}$ long) with small soft testicles $(<2 \mathrm{ml}$ volume) (figure). His sense of smell was normal, and his skeletal age was $9 \cdot 1$ years. Serum thyroxine, thyroid stimulating hormone, prolactin, and cortisol concentrations were all normal. A sequential insulin and arginine stimulation test after priming with $100 \mu \mathrm{g}$ ethinyloestradiol daily for three days confirmed growth hormone deficiency with unrecordable growth hormone concentrations despite adequate hypoglycaemia $(4.2 \mathrm{mmol} / \mathrm{l} \rightarrow 2 \mathrm{mmol} / \mathrm{l})$. The pituitary sella was small but a brain scan was normal. He was given growth hormone (Somatonorm) 4 units subcutaneously three times a week, and his height velocity (which had been $4.8 \mathrm{~cm} /$ year before treatment) increased to $10.8 \mathrm{~cm} /$ year during the first full year of treatment.

At the age of 14.3 years he was still prepubertal His gonadotrophin response to gonadotrophin releasing hormone was poor with a peak measurement of luteinising hormone of $3.1 \mathrm{IU} / \mathrm{l}$ and a peak measurement of follicle stimulating hormone of $2 \cdot 1$ IU/l. Basal testosterone concentration was $0 \cdot 1$ $\mathrm{nmol} / \mathrm{l}$. The addition of testosterone enanthate 100 $\mathrm{mg}$ intramuscularly monthly has accelerated his growth (which at the time of writing was 9.7 $\mathrm{cm} /$ year), and induced sexual maturation.

\section{Discussion}

A ring 18 chromosome karyotype is comparatively rare; there is loss of small distal segments of both long and short arms of the chromosome so that a mixture of physical signs characteristic of $18 \mathrm{p}-$ and 